\title{
Design of a fluorescence technique using double laser pulse excitation for the measurement of molecular Brownian dynamics
}

\author{
J.R. Torga ${ }^{\text {a, } *, 1}$, J.I. Etcheverry ${ }^{b}$, M.C. Marconi ${ }^{\text {a.2 }}$ \\ ${ }^{a}$ Departamento de Fisica, Facultad de Ciencias Exactas y Naturales, UBA, Ciudad Uniuersitaria, Pabellón 1, 1428 Buenos Aires, \\ Argentina \\ ${ }^{b}$ Departamento de Matemática, Facultad de Ciencias Exactas y Naturales, UBA, Ciudad Universitaria, Pabellón 1, 1428 Buenos Aires, \\ Argentina
}

Received 12 March 1997; revised 16 May 1997; accepted 9 June 1997

\begin{abstract}
A complete analysis of a novel method to evaluate the rotational diffusion time of fluorescent molecules is presented. It is based on the study of the total fluorescence produced after excitation with two consecutive short laser pulses. Expressions for the total fluorescence energy emitted in this case are derived, which would allow the evaluation of the rotational diffusion time. The main advantages of the proposed method are its simple setup, a time resolution only limited by the exciting laser pulsewidth, and the fact that it does not require fast time response detectors. 1997 Elsevier Science B.V.
\end{abstract}

PACS: $33.15 . \mathrm{Vb} ; 32.50 .+\mathrm{d} ; 42.62 .-\mathrm{b}$

Keywords: Rotational depolarization; Brownian dynamics; Fluorescence anisotropy

The study of the fluorescence anisotropy produced in a fluorescent sample is a very helpful method to obtain information about the rotational dynamics of fluorophores. This technique for example may be used to evaluate the rotational Brownian motion of molecules with fluorescent groups rigidly attached, thus obtaining information about the volume and shape of the carrier molecules, or can reveal if the fluorophore can rotate freely or if it is restricted in its angular displacement by the surrounding environment $[1,2]$.

When a pulse of linearly polarized light impinges a sample composed of absorbing molecules, the angular anisotropy imposed by the light electric field direction

\footnotetext{
* Corresponding author. E-mail: torga@df.uba.ar.

' Fellow from CONICET.

${ }^{2}$ Member of Consejo Nacional de Investigaciones Cientificas, CONICET.
}

produces a photo-selection in the sample inducing an anisotropy displayed by a non-isotropic polarization of the fluorescence. The degree of polarization of this signal can be measured under steady-state conditions using $\mathrm{CW}$ excitation light sources to obtain a mean value. However, the most important aspect of fluorescence anisotropy techniques are time-resolved studies, which allow the direct measurement of the rotational correlation times but require detectors faster than the characteristic decay times. A meaningful asset in this field was the utilization of ultrashort pulsed laser sources, that allow the investigation of processes with time resolutions in the picosecond range.

Extensive work has been done in the theoretical aspect by Perrin [3], Jablonski [4], Weber [5], Chuang et al. [6] and Tao [7], who derived expressions for the polarization of the fluorescence for different fluorophore geometries. A very comprehensive review in this area is the paper by Kawski [8]. Time-resolved rotational diffusion was also measured using stimulated Raman scattering [9] and anisotropic picosecond absorption $[10,11]$. 
In this paper we propose a novel method, that consists in the study of the total fluorescence produced when the sample is illuminated with two short laser pulses, as a function of the delay between the pulses. This approach has the advantages of a simple experimental set up and the possibility of obtaining rotational correlation times with a time resolution only limited by the excitation pulse-width.

Two linearly polarized short laser pulses with the same polarization are successively focused in a fluorescent sample, separated in time by a delay $t_{0}$. The first pulse produces a photo-selected ensemble of excited molecules along a direction in space defined by the light polarization. The consequent anisotropy changes the characteristics of the fluorescence (polarization and intensity) originated by the second laser pulse, as this pulse will find a non-homogeneously distributed ensemble of non-excited molecules, provided that the delay time between pulses $t_{0}$ is smaller than the fluorescence decay and rotation times. The fluorescence produced with the double pulse excitation is time integrated with a slow-time-response detector and is analyzed as a function of the delay between pulses. This has the advantage of not requiring fast detectors.

In this work we present a complete analysis of this proposed method to show how the rotational diffusion time can be retrieved from the total fluorescence measurements with two laser pulse excitation. In this calculation we assume spherical molecules and very short excitation pulses. We also present numerical simulations corresponding to non-spherical fluorophores and non-delta excitation pulses, and a feasibility analysis for a typical case.

After the excitation by the first pulse, the initial angular distribution of excited molecules changes in time due to Brownian rotational movement, then modifying the distribution of excited molecules and relaxing again to an isotropic distribution. Following Ref. [7], the angular distribution of excited molecules immediately after the excitation with the first delta pulse is given by:

$n_{1}(\theta, t=0)=n_{0}\left[1-\exp \left(K \cos ^{2}(\theta)\right)\right]$.

where $n_{1}(\theta, t=0)$ represents the density of excited dipoles at an angle $\theta$ relative to the polarization axis of the exciting pulse, and $n_{0}$ is the initial isotropic population of absorbing molecules. $K=I \sigma / h \nu$ is a factor that accounts for the fraction of molecules excited in the sample and can be calculated from the total energy of the excitation pulse $I$ and the absorption cross section of the fluorescent molecule $\sigma$. For example, for typical values $\sigma=10^{-16}$ $\mathrm{cm}^{2}$ and $\lambda=570 \mathrm{~nm}$, an intensity of $3.4 \times 10^{-4} \mathrm{~J} / \mathrm{cm}^{2}$ is necessary to achieve $K=0.1$.

We describe the population of excited fluorophores $n(\theta, \phi, t)$ in the usual way $[6,8]$. In the case of spherical molecules, the rotational diffusion term reduces to a diffusion constant $D$ times the angular part of the Laplacian of the molecular density, and the whole problem has rota- tional symmetry in the direction of the excitation laser polarization. Accordingly, we have:

$$
\begin{aligned}
\frac{\partial n(\theta, t)}{\partial t}= & K I(t) \cos ^{2}(\theta)\left[n_{0}-n(\theta, t)\right]-\frac{n(\theta, t)}{\tau} \\
& +D \Delta_{H, \varphi} n(\theta, t),
\end{aligned}
$$

where $\tau$ is the characteristic fluorescence time.

As we assume delta pulse excitations, the evolution of the population of excited molecules away from the excitation pulses is given only by the diffusion and fluorescence decay terms. Under these assumptions, and using the linearity of the problem, it is easy to find a general expression for the density of molecules excited by the first and the second laser pulses, $n_{1}$ and $n_{2}$, respectively, in terms of the eigenfunctions $\Psi_{j}(\theta)$ of the rotational diffusion operator:

$$
\begin{aligned}
n_{i}(\theta, t)= & n_{0} \exp \left(\frac{t-t_{i}}{\tau}\right) K \sum_{j} c_{j}^{(i)} \Psi_{j}(\theta) \\
& \times \exp \left(E_{j}\left(t-t_{i}\right)\right), \quad i=1,2 .
\end{aligned}
$$

$E_{j}$ are the eigenvalues corresponding to $\Psi_{j}(\theta), c_{j}^{(i)}$ are constants to be determined in order to satisfy adequate initial data, and $t_{i}, i=1,2$, represent the arrival time of each laser pulse, $t_{1}=0$ and $t_{2}=t_{0}$. In the particular case we are considering, the orthogonal eigenfunctions are the Legendre polynomials, $\Psi_{j}(\theta)=A_{j} P_{j}(\cos (\theta))$, where $A_{j}$ is a normalization factor and the corresponding eigenvalues are $E_{j}=j(j+1) D$.

With this formalism, we obtain the density of excited molecules due to the first laser pulse, using (1) as initial data. Developing this expression up to second order in the excitation $K$ we have

$$
\begin{aligned}
n_{1}(\theta, t)= & n_{0} \exp (-t / \tau) K\left[\frac{1}{3}-\frac{1}{10} K+\left(\frac{2}{3}-\frac{2}{7} K\right)\right. \\
& \times P_{2}(\cos (\theta)) \exp (-t / \phi) \\
& \left.-\frac{4}{35} K P_{4}(\cos \theta) \exp (-10 t / 3 \phi)\right] .
\end{aligned}
$$

Here $\tau$ is again the fluorescence decay time and $\phi$ is the characteristic rotational diffusion time that, for a spherical rotor, is related to the diffusion coefficient $D$ through $\phi=1 / 6 D$.

After a delay $t=t_{0}$, the second pulse arrives to the sample. At this time, the distribution of non-excited molecules is no longer homogeneous, provided that the time delay between pulses is smaller than the fluorescence decay time and the rotational diffusion time. The angular distribution of excited dipoles at the arrival of the second pulse is given by $n_{1}\left(\theta, t_{0}\right)$. The density of absorbing molecules at this time will consequently be given by $n_{i}\left(\theta, t_{0}\right)=n_{0}-n_{1}\left(\theta, t_{0}\right)$. Following the derivation of Eq. (1), the density of the molecules excited by the second laser pulse is obtained as

$n_{2}\left(\theta . t_{0}\right)=\left[n_{0}-n_{1}\left(\theta . t_{0}\right)\right]\left[1-\exp \left(K \cos ^{2}(\theta)\right)\right]$. 
The expression for $n_{2}(\theta, t)$ for $t>t_{0}$, obtained according to Eq. (3) with initial condition given by (5), and considering only up to second order in $K$, is

$$
\begin{aligned}
n_{2}(\theta, t)= & C_{0}+C_{2} P_{2}(\cos (\theta)) \exp \left(-\frac{t-t_{0}}{\phi}\right) \\
& +C_{4} P_{4}(\cos (\theta)) \exp \left(-\frac{10}{3} \frac{t-t_{0}}{\phi}\right),
\end{aligned}
$$

where the coefficients $C_{j}$ are given by

$$
\begin{aligned}
C_{0}= & n_{0} K\left[\frac{1}{3}-\frac{1}{10} K-\frac{4}{45} K \exp \left(-t_{0}(1 / \tau+1 / \phi)\right)\right], \\
C_{2}= & n_{0} K\left[\frac{2}{3}-\frac{2}{7} K-\frac{2}{9} K \exp \left(-t_{0} / \tau\right)\right. \\
& \left.-\frac{22}{63} K \exp \left(-t_{0}(1 / \tau+1 / \phi)\right)\right], \\
C_{4}= & n_{0} K\left[-\frac{4}{35} K-\frac{8}{3.5} K \exp \left(-t_{0}(1 / \tau+1 / \phi)\right)\right] .
\end{aligned}
$$

The total fluorescence intensity produced by each pulse. with polarizations in the direction perpendicular $\left(I_{r, i}(t)\right)$ and parallel $\left(I_{z . i}(t)\right)$ to the polarization of the exciting pulse are given by

$$
\begin{aligned}
& I_{z, i}(t)=h \nu \int \frac{n_{i}(\theta, t)}{\tau} \cos ^{2}(\theta) \mathrm{d} \Omega, \\
& I_{y, i}(t)=h \nu \int \frac{n_{i}(\theta, t)}{\tau} \sin ^{2}(\theta) \sin ^{2}(\varphi) \mathrm{d} \Omega .
\end{aligned}
$$

After integration in time, the contribution of the $i$ th pulse to the total fluorescence energy emitted in both polarizations is finally obtained:

$$
\begin{aligned}
E_{:, i}\left(\tau, \phi, t_{0}\right)= & h \nu \int_{t i}^{x} \int_{\Omega} \frac{n_{i}(\theta, t)}{\tau} \cos ^{2}(\theta) \mathrm{d} \Omega \mathrm{d} t, \\
E_{y, i}\left(\tau, \phi, t_{0}\right)= & h \nu \int_{t i}^{\infty} \int_{\Omega} \frac{n_{i}(\theta, t)}{\tau} \\
& \times \sin ^{2}(\theta) \sin ^{2}(\varphi) \mathrm{d} \Omega \mathrm{d} t,
\end{aligned}
$$

where we have explicitly shown the dependence of the total fluorescence energy on the fluorescence time, on the diffusion time, and on the time delay between pulses. The total fluorescence energy with each polarization is then obtained by summing the contributions of each pulse:

$$
\begin{aligned}
E_{z}\left(\tau, \phi, t_{0}\right)= & \frac{h \nu}{\tau} \int_{\Omega}\left(\int_{0}^{\infty} n_{1}(\theta, t) \mathrm{d} t+\right. \\
& \left.\int_{t 0}^{\infty} n_{2}(\theta, t) \mathrm{d} t\right) \cos ^{2}(\theta) \mathrm{d} \Omega, \\
E_{y}\left(\tau, \phi, t_{0}\right)= & \frac{h \nu}{\tau} \int_{\Omega}\left(\int_{0}^{\infty} n_{1}(\theta, t) \mathrm{d} t\right. \\
& \left.+\int_{t 0}^{\infty} n_{2}(\theta, t) \mathrm{d} t\right) \sin ^{2}(\theta) \sin ^{2}(\varphi) \mathrm{d} \Omega
\end{aligned}
$$
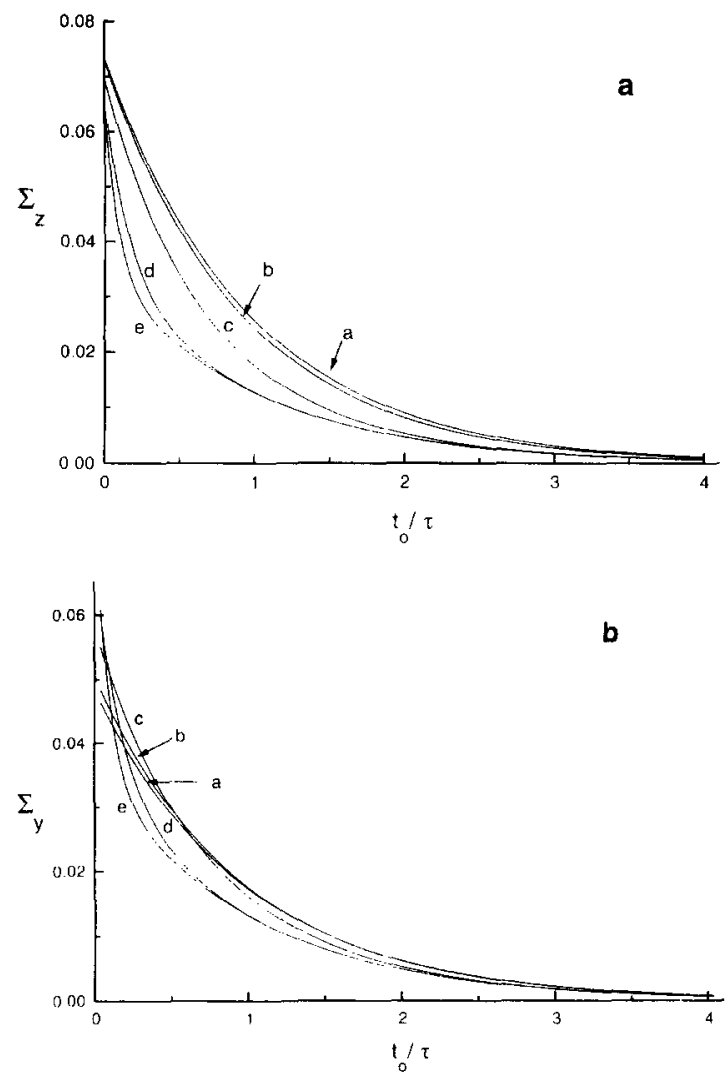

Fig. 1. Differential fluorescence signal (a) parallel $\left(\Sigma_{z}\right)$ and (b) perpendicular $\left(\Sigma_{v}\right)$ to the excitation direction as a function of the delay time between pulses for different values of the rotation time. $\mathrm{a}: \phi / \tau=10, \mathrm{~b}: \phi / \tau=5, \mathrm{c}: \phi / \tau=1, \mathrm{~d}: \phi / \tau=1 / 5, \mathrm{e}: \phi / \tau$ $=1 / 10$. Times are normalized to the fluorescence decay time. $K=0.1$.

The method we are discussing in this paper consists of the measurement of the total fluorescence when two pulses excite the sample, as a function of the time delay $t_{0}$. The total fluorescence produced under these conditions is compared with the fluorescence that would be produced in the case of two very delayed pulses. The difference is due to the fact that the second pulse arrives to the sample when the anisotropy in the angular distribution of non-excited molecules induced by the first pulse is still present. Accordingly, we can define a parameter of interest, the differential fluorescence signal $\Sigma$ for both polarizations, as:

$$
\Sigma_{k}\left(\tau, \phi, t_{0}\right)=-\frac{E_{k}\left(\tau, \phi, t_{0}\right)-E_{k}(\tau, \phi,+\infty)}{E_{k}(\tau, \phi,+\infty)}, \quad k=y, z
$$



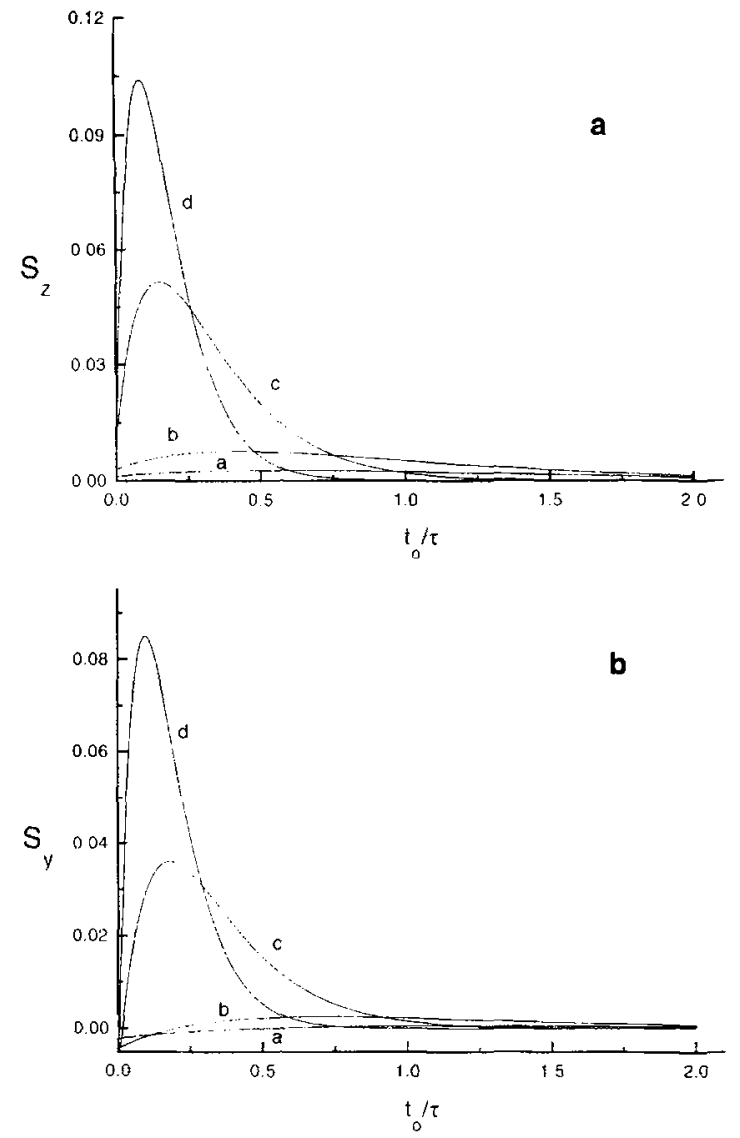

Fig. 2. Sensitivity $S=\partial \Sigma / \partial \phi$. (a) parallel $\left(S_{.}\right)$and (b) perpendicular $\left(S_{v}\right)$ to the excitation direction, as a function of the time delay between pulses. for different values of the rotational diffusion time: $\mathrm{a}: \phi / \tau=2 . \mathrm{b}: \phi / \tau=1, \mathrm{c}: \phi / \tau=1 / 5, \mathrm{~d}: \phi / \tau=1 / 10$. Times are normalized to the fluorescence decay time. $K=0.1$.

The explicit expressions obtained from (14) and (15) for the spherical molecules case are

$$
\begin{aligned}
& \Sigma_{(}\left(\tau, \phi . t_{1}\right) \\
&=K \exp \left(-t_{0} / \tau\right) \frac{\tau}{\tau+\phi} \\
& \quad \times \frac{\frac{1}{5} \phi+\frac{1}{9} \tau+\frac{8}{35} \exp \left(-t_{0} / \phi\right) \phi+\frac{4}{45} \tau \exp \left(-t_{0} / \phi\right)}{\left(\frac{1}{3}-\frac{1}{10} K\right) \tau+\left(\frac{4}{15}-\frac{4}{35} K\right) \tau \phi /(\tau+\phi)}, \\
& \Sigma_{y}\left(\tau, \phi, t_{0}\right) \\
&= K \exp \left(-t_{0} / \tau\right) \frac{\tau}{\phi+\tau} \\
& \quad \times \frac{\frac{1}{15} \phi+\frac{1}{9} \tau+\frac{2}{105} \exp \left(-t_{0} / \phi\right) \phi+\frac{4}{45} \tau \exp \left(-t_{0} / \phi\right)}{\left(\frac{1}{3}-\frac{1}{10} K\right) \tau-\left(\frac{2}{15}-\frac{2}{35} K\right) \tau \phi /(\tau+\phi)} .
\end{aligned}
$$

This differential signal depends on the fraction of excited molecules, the time delay $t_{0}$, and the decay times $\tau$ and $\phi$. In consequence. knowing the fluorescence decay time and calculating the fraction of excited molecules in the sample. it is possible to evaluate the rotation time $\phi$ from the measurement of the dependence of $\Sigma$ on the time delay $t_{0}$.

As a typical example, let us consider rhodamine $6 \mathrm{G}$ in ethylene glycol at $290 \mathrm{~K}$, with fluorescence and rotational diffusion times both of about $4 \mathrm{~ns}$ [8]. We consider an excitation pulse of $10 \mathrm{~nJ}$, centered at $\lambda=570 \mathrm{~nm}$, focused to $100 \mu \mathrm{m}$ diameter in a sample $1 \mathrm{~mm}$ long with $10^{-5}$ molar concentration. Assuming an absorption cross section $\sigma=10^{-16} \mathrm{~cm}^{2}$, we obtain $K=0.014$, which corresponds to a fraction of excited molecules of $0.5 \%$. According to (17). (18), and assuming a collecting efficiency of $10 \%$, the differential energy would be $10^{-14} \mathrm{~J}$, with a total fluorescence energy of $10^{-12} \mathrm{~J}$. This difference can be detected averaging the signal using a high repetition rate laser excitation.

Fig. 1 shows plots of expressions (17) and (18) as a function of the time delay $t_{0}$ normalized to the fluorescence decay time $\tau$. for different ratios $\phi / \tau$. For fluores-
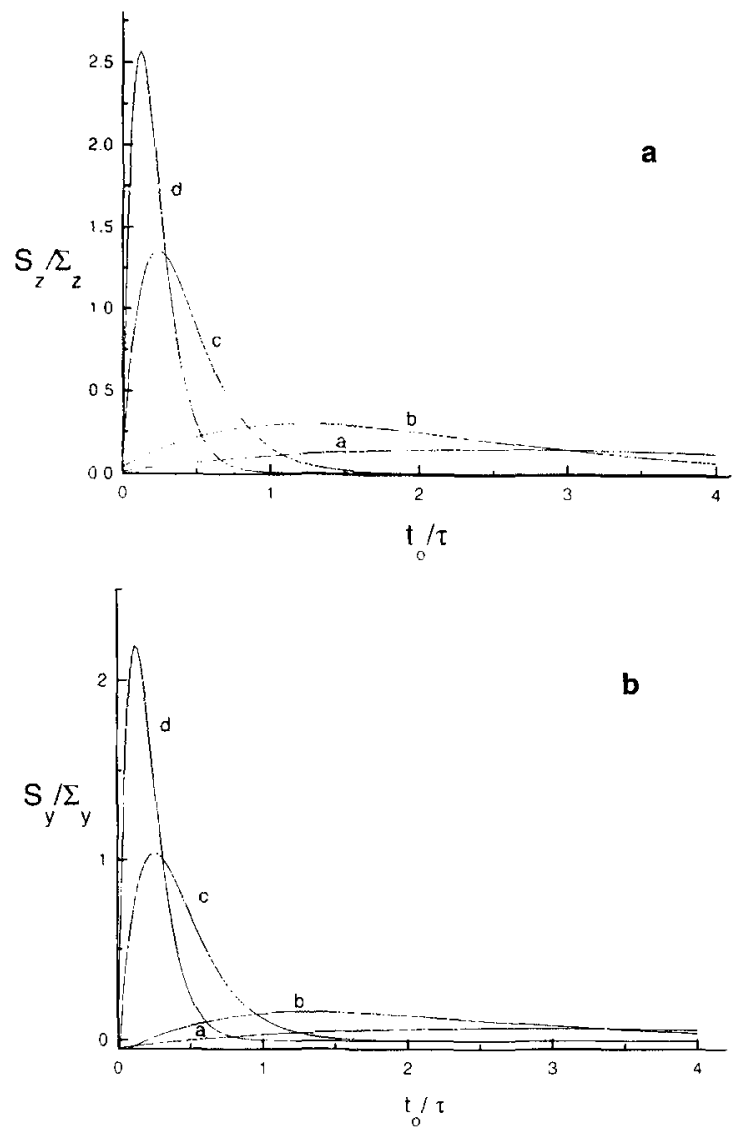

Fig. 3. Normalized sensitivity $S / \Sigma$, (a) parallel $(S, \Sigma)$ and (b) perpendicular $\left(S_{y} / \Sigma\right)$ to the excitation direction, as a function the time delay between pulses, for different values of the rotational diffusion time. a: $\phi / \tau=2 . \mathrm{b}: \phi / \tau=1, \mathrm{c}: \phi / \tau=1 / 5, \mathrm{~d}$ : $\phi / \tau=1 / 10 . K=0.1$. 
cence times small compared with $\phi$, the molecule will emit before a significant rotation takes place. In these conditions the differential fluorescence signal is large, and the effect of the double excitation is important for time delays up to values of the order of $\tau$. If the fluorescence time is large compared with the rotation time, the dipole will significantly rotate before de-excitation by fluorescence. Under these conditions the differential signal is only noticeable for short delays up to values of the order of $\phi$.

Notice that for different ratios $\phi / \tau$, the differential fluorescence curves are more sensitive as $\phi / \tau$ decreases. For example, changing the value of $\phi / \tau$ from 5 to 10 , produces a maximum change in the differential fluorescence signal of $6 \%$, while $\Sigma$ changes about $18 \%$ when $\phi / \tau$ varies from $1 / 5$ to $1 / 10$.

This can be easily seen in Fig. 2 where the sensitivity $S=\partial \Sigma / \partial \phi$ is plotted as a function of $t_{0} / \tau$ for different values of $\phi / \tau$. The figure shows that for delay times longer than $\tau$, the technique will not be useful because all curves go to zero. This is expected from the fact that information is retrieved from the fluorescence signal, that goes to zero for $t_{0} \gg \tau$.

In order to evaluate the range of $\phi / \tau$ values where this method will be useful, we plot in Fig. 3 the normalized sensitivity $S / \Sigma$, as a function of $t_{0} / \tau$ for different ratios $\phi / \tau$. The curves show that maximum sensitivity is obtained for delay times $t_{0}$ of the order of $\phi$. All the plots shown correspond to a fraction of excited molecules of $3 \%$.

From the two previous figures, we conclude that the three times involved $t_{0}, \tau, \phi$, should be of about the same order to obtain good signal and sensitivity.

The same behavior for the differential energy $\Sigma$ is obtained if we consider finite pulse width excitation. Fig. 4

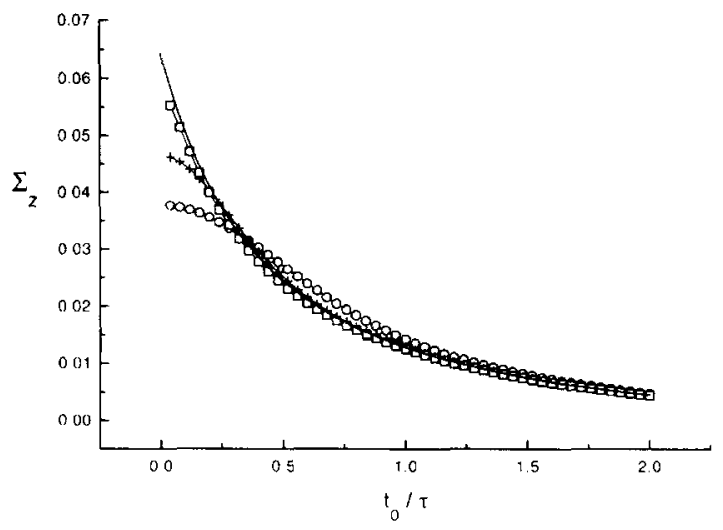

Fig. 4. Differential fluorescence signal parallel $\left(\Sigma_{.}\right)$to the excitation direction as a function of the delay time between pulses for Gaussian excitation pulses of widths (FWHM): $\tau / 2$ (circles), $\tau / 4$ (crosses), $\tau / 16$ (squares). Times are normalized to the fluorescence decay time, and $\phi=\tau$. Solid line: delta excitation. shows the numerical results for Gaussian excitation pulses with $\tau / 2, \tau / 4$, and $\tau / 16$ FWHM. The convergence of the numerical results for decreasing pulse width to the delta excitation results is clearly observed. Particularly, for $t_{0} \leq$ $\tau / 20$ the differences between the two results are negligible.

In conclusion, we have presented a complete analysis of a proposed alternative method that allows the evaluation of the ratio between the rotational diffusion time and the fluorescence decay time for fluorescent molecules, using double laser pulse excitation. As a representative example, the calculations for the simple case of a spherical rotor are presented, but this formalism can be applied to other fluorophore geometries. In particular, the case of symmetric top molecules with the excitation dipole along the main symmetry axis has the same formal expressions, where the diffusion constant $D$ is the corresponding element of the diffusion tensor along the symmetry axis. Numerical calculations for finite pulse width excitation are also presented, showing that for realistic pulse widths the expected signal is very close to the analytical results for delta pulse excitation.

An interesting feature of the proposed method is that it does not require a fast detection system, and the information is retrieved from the time integrated fluorescence signal. Resolution is only limited by the pulse width of the laser excitation, although better signal and sensitivity are obtained when $t_{0}, \tau, \phi$ are all of about the same order.

\section{Acknowledgements}

This work was supported by the Universidad de Buenos Aires (PMA Program and grant EX285) and a grant from Fundación Antorchas. We thank P.F. Aramendia and M. Negri for helpful discussions on several aspects of this work.

\section{References}

[1] J.R. Lakowicz, Principles of Fluorescence Spectroscopy (Plenum, New York, 1986)

[2] F. Perrin, Ann. Phys. 12 (1929) 169.

[3] F. Perrin, Acta Phys. Pol. 5 (1936) 335.

[4] A. Jablonski, Bull. Acad. Pol. Sci. 8 (1960) 259.

[5] G. Weber, J. Phys. Chem. 93 (1989) 6069.

[6] T.J. Chuang, B.K. Eisenthal, J. Chem. Phys. 57 (1972) 5094

[7] T. Tao, Biopolymers 8 (1969) 609.

[8] A. Kawski, Crit. Rev. Anal. Chem. 23 (1993) 459.

[9] E.A.J. Spaans, M.P. van Exter, A. Lagendijk. Optics Comm. $76(1990) 376$.

[10] C.V. Shank, E.P. Ippen, Appl. Phys. Lett. 26 (1975) 62.

[11] D.H. Waldeck, G.R. Fleming, J. Phys. Chem. 85 (1981) 2614 